\title{
FROM PARENTHOOD TO TUTTO PUÒ SUCCEDERE
}

\author{
READY-MADE ELEMENTS AND CULTURAL \\ TRANSLATION
}

\author{
Stefania Antonioni \\ Università di Urbino \\ stefania.antonioni@uniurb.it \\ Chiara Checcaglini \\ Università di Udine \\ chiara.checcaglini@uniud.it
}

\begin{abstract}
In our article we propose an analysis of the Italian TV series Tutto Può Succedere (2015-2018), a remake of the American TV series Parenthood (2010-2015). The Italian remake precisely reproduces some Parenthood's ready-made elements: the two series share the main plot, many storylines and characters' personalities, dealing with the ups and downs of a large family, formed of four siblings, their parents and their children. We will focus on the differences and similarities of the two shows from several perspectives, such as formal and content divergences, and their cultural, social and production implications. On the one hand, the Italian remake loses the distinguishing style and the faster pace of the original series to adjust to Rai's more basic aesthetics; on the other hand, Rai chooses to focus on specific plots and characters that match its own purposes. Indeed, we can assume that the youngest characters are the means by which Raiuno tries to connect with younger viewers, and the same function is assumed by the role played by music in the series and by the on-screen presence of young Italian musicians.
\end{abstract}

Keywords: cultural translation, remake, adaptation, serial narratives, Parenthood, Tutto Può Succedere

\section{Introduction: Format Remake, Format Adaptation, Format Translation and the Amount of Ready-Made}

Our article analyses the Italian TV series Tutto Può Succedere (2015-2018), which is an outstanding case in the Italian television panorama, being the first remake by the Italian Public Broadcasting Service of an American TV show, Parenthood. It aired on Raiuno, the main TV channel of Italian PBS Rai, known as the most conservative and mainstream among Rai channels. 
Tutto Può Succedere is not the first Italian remake of a foreign TV show: there are many successful examples of Italian adaptations of formats or series, such as, just to quote a few, I Cesaroni (Mediaset), adapted from the Spanish series Los Serrano, Raccontami (Rai) inspired by Cuéntame Còmo Pasò, Braccialetti Rossi (Rai) from the Catalan format Polseres Vermelles, Un Medico in Famiglia (Rai) adapted from Médico de Familla.

It is worth noting that the aforementioned series are all Spanish productions and formats: the recurring presence of Spanish adaptations in Italian programming could be explained by the audience (and broadcasters) perception of a common cultural ground shared by Italy and Spain. On the contrary, Tutto Può Succedere stands as a different case, being the first Rai remake that confronts the style, the innovations and the achievements of 2000s U.S. serial storytelling.

We will argue that Tutto Può Succedere represents a peculiar case of a remake that intertwines a ready-made approach, imitating Parenthood storylines, characters, even the spatial structure of the set, with culturally defined elements, such as locations, and specific choices aimed to appeal to younger generations. We will assert that the peculiar focus of the storylines on the younger characters and the soundtrack, that picks from contemporary indie Italian music, are part of Raiuno's strategy to refresh its brand image and, in the meantime, to widen and rejuvenate its audience.

Before starting our analysis, it is necessary to illustrate our theoretical basis, which are clearly rooted in the field of format studies stream, widened and well established in recent years. Even though our aim is not to recall the history of television content production and distribution, it is useful to evoke Albert Moran's pivotal distinction between canned and format programmes. The former, appearing at first in US network television at the end of the 1940s and early 1950s, refers to a type of programme that "has been broadcast and recorded for transmission in another time and place", ${ }^{1}$ whose international circulation begun in the "1950s thanks to dubbing and subtitling. As for the latter, "a TV format is a complex, coherent package of industry knowledge that is licensed to facilitate the making of another version of program in another television market. Thus, the devising and development of the program can occur in one place before a package of know-how is assembled, so that the program can be put together again in another territory". ${ }^{2}$

The 1990s were a watershed for both categories of programmes: the production of the canned ones changed, and their distribution could no longer be described as a one-way from the US to the rest of the world. On the other hand, at the end of the 1990s, three of the most successful and popular formats aired and began circulating worldwide: Who Wants to Be a Millionaire?, Survivor and Big Brother. Particularly, as underlined by Mats Nylund, "the rise of the format industry is related to several changes in the television industry in the last 20 years. Digitalization, globalization and commercialization come across as key tendencies". ${ }^{3}$ As for several decades the mostly commercialized format genre was the game show, at the turn of the millennium it was the turn of factual shows; only in recent years scripted formats became booming business. ${ }^{4}$ The late development of drama genre formatting is due to the complexity of content that requires an attentive work of cultural adaptation, not to mention its expensiveness and riskiness. Therefore, the reactualisation of scripted formats for new de-territorialised audiences must be particularly sensitive to local viewers' culture, values, ideals and even tastes. According to Albert Moran and Karina Aveyard's metaphor, a format can be seen as a template, in the sense that it "allows the programme to be adapted and produced for broadcast in other territories"; at the same time "these templates are also flexible - capable of being moulded to suit the particular social inflexion and cultural nuances of the broadcaster and territory for which it is remade". ${ }^{5}$ In other words, elements of the template can be considered ready-made parts of the narrative, reproducible in other cultures, while other elements must be adapted and customised to become familiar to different audiences, and rooted in a given territory.

This swinging between dichotomies gave rise to format glocalisation, indigenisation, domestication, but also to a parochial internationalism ${ }^{6}$ or to translocalisation ${ }^{7}$ all concepts underlined by different theoretical approaches, and it is made clear that this type of programme tries to integrate universal elements (that are repeated) and peculiar traits (that are locally and continuously replaced). Therefore, we can assume that "when a television series is remade in another country, the universal elements of that format will interact and dialogue with the 
local factors that arise from its new local context [...] all of its elements must be transformed according to the defining coordinates (whether they are historical, sociocultural, political or related to the broadcasting industry) of this new context". ${ }^{8}$

Among the universal elements that can make a scripted format successful, we find the sense of "cultural proximity" underlined by Joseph Straubhaar, ${ }^{9}$ namely the characteristics reflecting the traditional, regional and cultural values of a people, usually applied in cultural translation. Subsequently, the same author widens his understanding of cultural proximity, stating that common values, images, archetypes and themes across cultures allow programmes to flow beyond cultural boundaries. ${ }^{10}$ This type of cultural proximity is consistent with what can be called the universal, ready-made factors that are saved and retained in the process of cultural translation.

Another key element assuring the true success of a TV series remake is its transparency, that is to say "the capability of certain texts to seem familiar regardless of their origin, to seem a part of one's own culture, even though they have been crafted elsewhere". ${ }^{11}$ Therefore, the quality of narrative transparency can be leveraged by certain poignant global formats in their journey across different markets.

Coherent with this process of cultural continuity is the adaptation following the so called "transposed narrative" strategy, where "narrative structure, content and sequencing remain largely unchanged, with modifications merely to certain aspects of the story to enhance cultural proximity, such as location, actors, cultural, institutional and historical references". ${ }^{12}$ As we will see in the next paragraphs, our case study Tutto Può Succedere mostly followed a transposed narrative strategy, even though some changes were needed to adapt the series to the perceived Italian cultural context, as we will outline thereafter.

\section{Parenthood and Tutto Può Succedere: Ready-made Elements and Transposed Narrative}

Parenthood is an American TV series developed by Jason Katims, loosely based on the 1989 film of the same name, directed by Ron Howard, and its television adaptation as a sitcom in 1990. Parenthood's six seasons aired on NBC from 2010 to 2015, with each season presenting thirteen to twenty-two 40-minutes episodes.

Tutto Può Succedere is produced by Cattleya and Rai Fiction and aired on Raiuno. Its first season aired from December 27, 2015 to March 13, 2016, its second season aired from April 20 to June 29, 2017, and its third and final season aired from June 18 to August 6, 2018. Parenthood revolves around the ups and downs of the large Braverman family, which becomes the Ferraro family in the Italian remake. The two series share the main plot, many storylines and characters' personalities, dealing with a variety of problems, issues, conflicts among the family members, formed of four siblings, their parents and their children. The series especially focuses on the intergenerational exchanges and clashes among grandparents, parents and siblings.

While the composition of the family is the same, the location and the context are obviously different: Parenthood is set in Berkeley, California, Tutto Può Succedere is set in Rome.

\section{Tutto Può Succedere website reads:}

Tutto Può Succedere is the first Raiuno remake of an American TV series. It is an Italian adaptation of Parenthood, created by Jason Katims and aired successfully on NBC for five years. The Italian setting required a remarkable rewriting, that tried to preserve the energy, the pace and the modernity/Zeitgeist of the original, thanks to a brilliant team of actors and the direction by Lucio Pellegrini. ${ }^{13}$ 
This summary underlines a conscious attempt to throw a connection with American quality television, recalling some features specifically linked to the original series - being captivating, innovative, connected to the present - but it also highlights the reworking effort that differentiates Tutto Può Succedere from Parenthood.

Building on Edward Larkey et alii definition previously quoted, we will argue that Tutto Può Succedere is a case of transposed narrative, because the main narrative structure, content, and sequencing mimic the original one, and the adjustments concern only a few aspects of the story, such as location, actors, cultural and social references. ${ }^{14}$

Unlike television formats, which are "systematically and consciously assembled to facilitate the future adaptation", ${ }^{15}$ the possibility of a remake is not necessarily expected, nor prepared. When a remake like Tutto Può Succedere occurs, it activates the ready-made potential of the original narrative elements. On the one hand Tutto Può Succedere mimics the original in many ways: plots and subplots are the same, as well as the sequence of events and the characters' design. On the other hand some details significantly change, following the need of both a cultural and a production-distribution adjustment.

The most striking similarities concern some basic elements of the series. First, the characters' names are translated whenever possible: Sarah (Lauren Graham) and Sara (Maya Sansa), Kristina (Monica Potter) and Cristina (Camilla Filippi), Julia (Erika Christensen) and Giulia (Ana Caterina Morariu), Max (Max Burkholder) and Max (Roberto Nocchi), Amber (Mae Whitman) and Ambra (Matilda De Angelis). Second, the opening titles sequence uses the same visual and conceptual device of the original: images of the actors and characters are superimposed on real photographs from the actors' childhood, blurring the boundaries between fiction and reality, echoing the themes key to the series, like family relationships, youth, adulthood and the flowing of life from childhood to old age. It also highlights the emotional involvement sought by the two series.
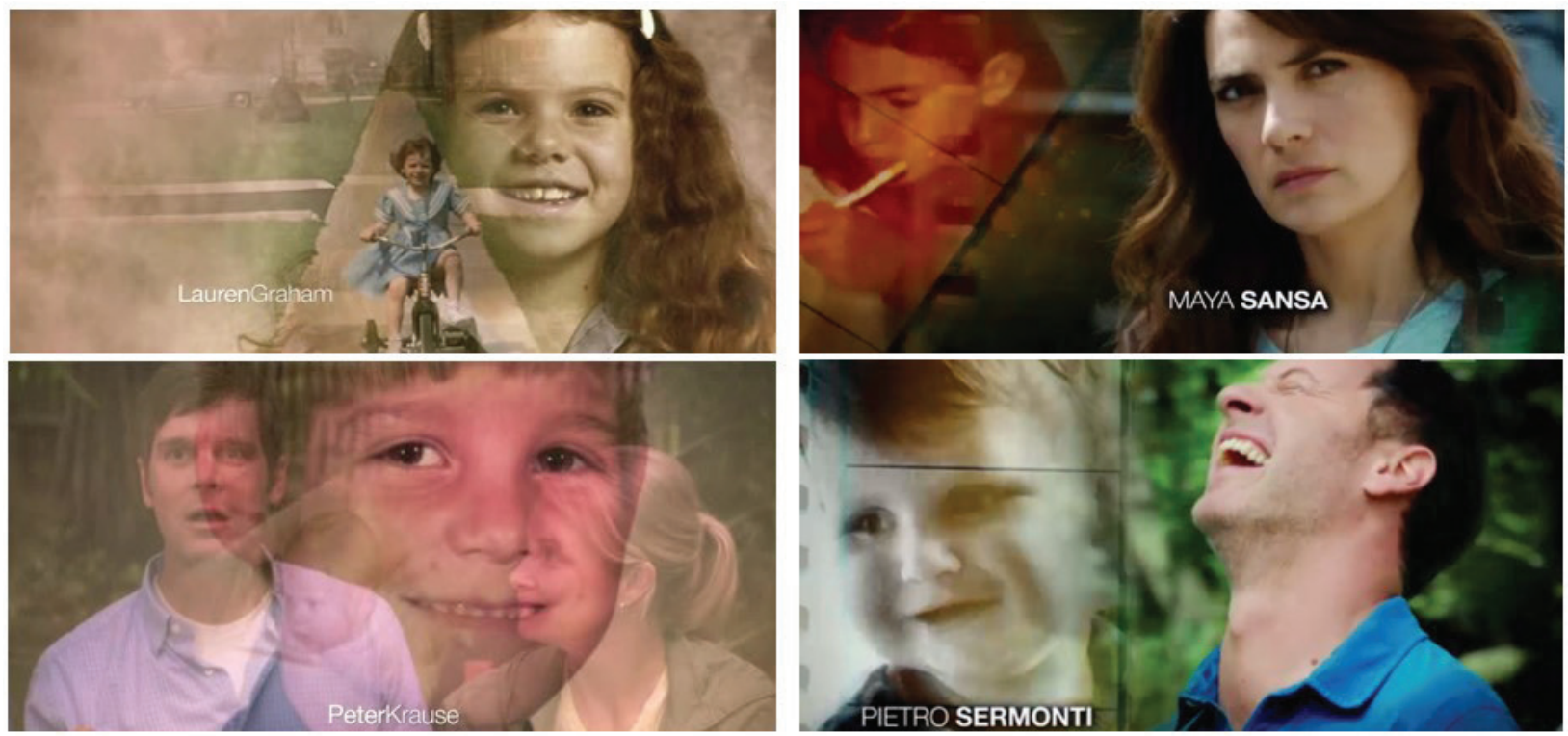

Figure 1. Parenthood (left) and Tutto Può Succedere's (right) opening titles.

The episode structure is also the same, even though apparently the two series differ in episode length, 45 minutes for each Parenthood episode, 100 minutes for Tutto Può Succedere's. However, the Italian remake merges more episodes at a time, according to the TV scheduling of Raiuno prime time, based on two hours slots (ads included). 
First, we will explore the narrative world of Tutto Può Succedere dwelling on settings and characters comparison. Second, we will analyse the different approach to the topics addressed by the series, ultimately focusing on Tutto Può Succedere distinguishing marks.

\subsection{Narrative Comparisons and Inconsistencies}

As we mentioned above, the action takes place in Rome, specifically in the neighbourhood of Fiumicino, which is a completely different location compared to Berkeley, California. However, the authors tried to reproduce the symbolic value of the original settings and spaces. Sometimes the adapted space works thanks to a cultural correspondence of the use and purpose of the set. The big grandparents' house represent the connection point for all the family members: a messy, but comforting environment, with a spacious green kitchen (the same color of Parenthood grandparents' kitchen), a patio full of plants and vegetation. The ritual Bravermans gatherings around the table to eat all together adapts particularly well to the Italian tradition of family lunches and dinners, therefore, unsurprisingly, such moments keep their dramatic purpose conveying a range of emotions, from convivial, joyful occasions to misunderstandings and outbursts of rage.
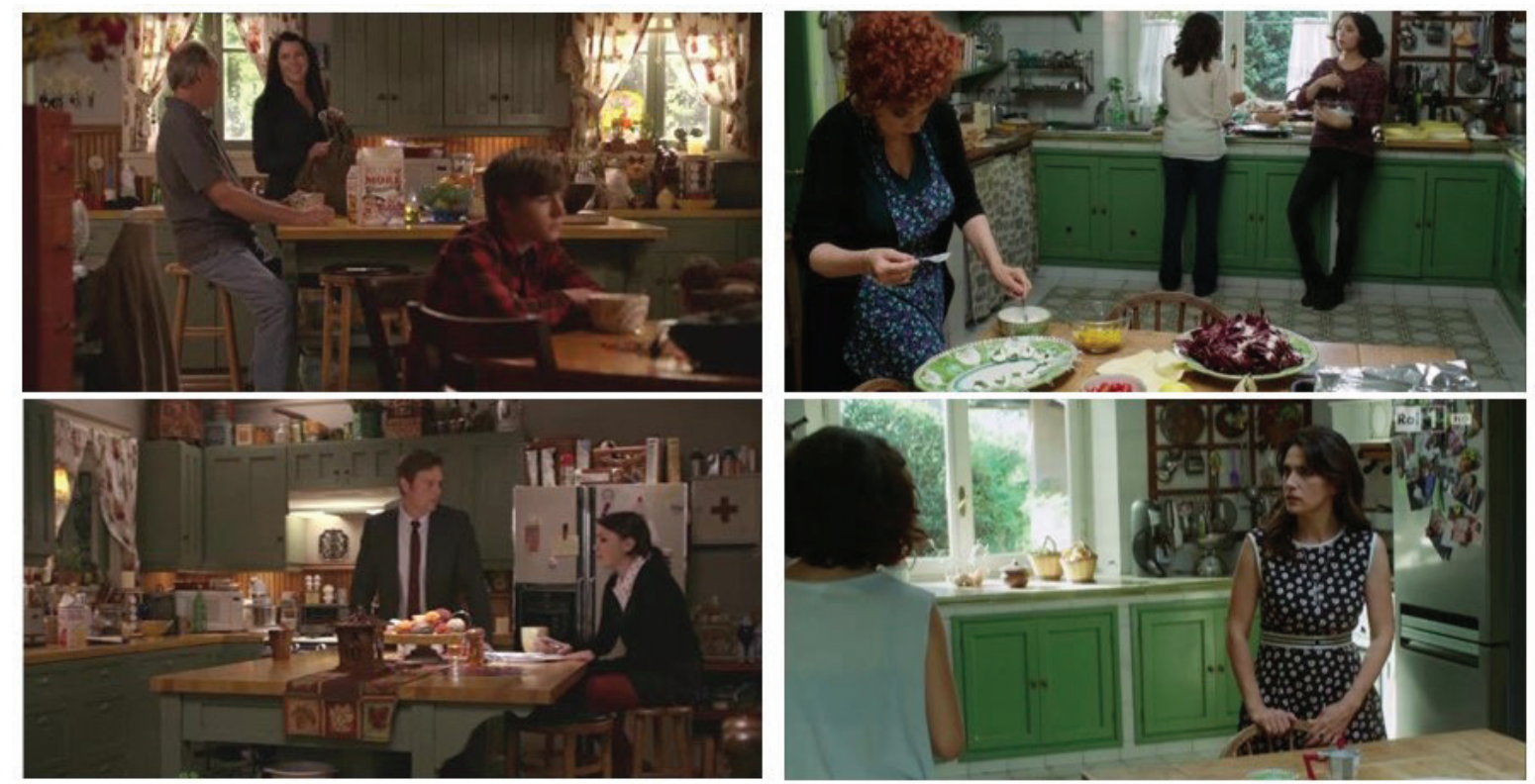

Figure 2. The green kitchen.

Other times the repurposing looks a bit more forced. Both younger brothers Crosby (Dax Shepard) and Carlo (Alessandro Tiberi) in the first seasons live on a boat; in Carlo's case, it is a floating house with a bar underneath, serving as both his home and his workplace. 

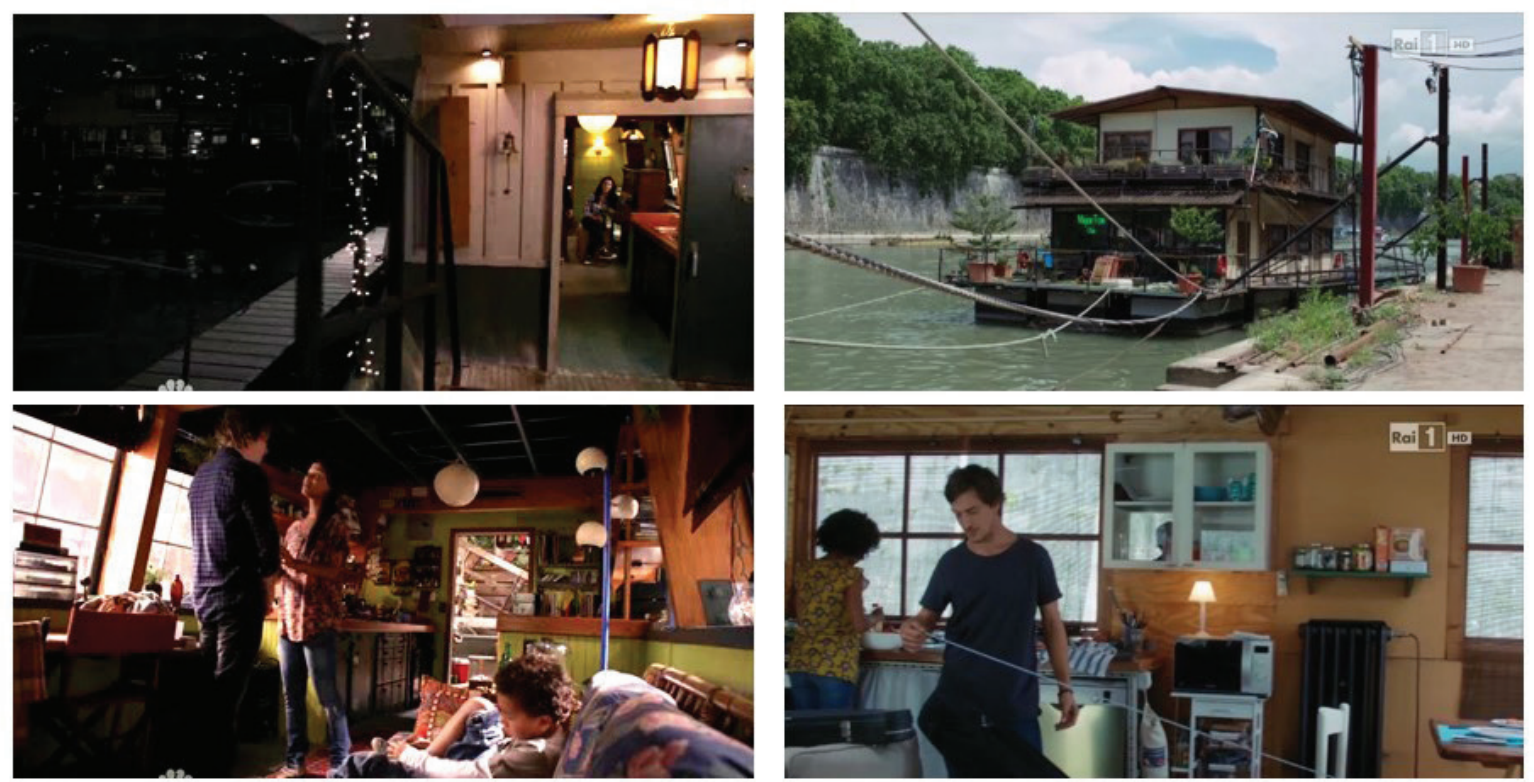

Figure 3. Crosby's (left) and Carlo's (right) houseboats.

Although it's not impossible that someone would live on a boat on the Tevere river, it still seems more unlikely than in Crosby's Berkeley houseboat. Other inconsistencies concern secondary details in characterisation: older brother Adam (Peter Krause) usually surfs in the ocean when he needs a break from everyday problems and stress. It seems much less plausible when Alessandro (Pietro Sermonti) does the same thing in the Tyrrhenian Sea.
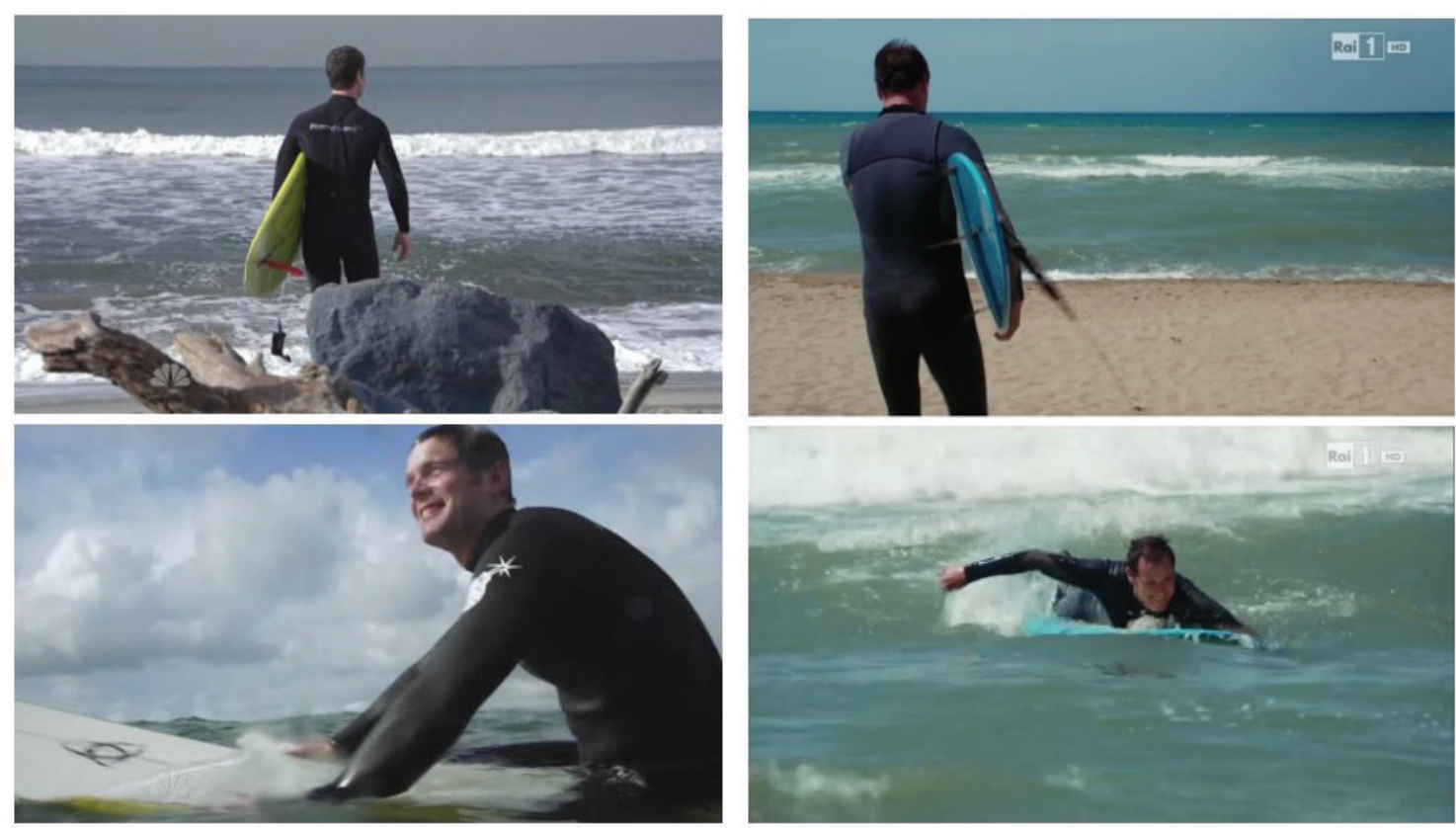

Figure 4. Surfing in the Ocean (left) and in the Tyrrhenian Sea (right). 
As for characters' look and attitude, the grandmothers Camille (Bonnie Bedelia) and Emma (Licia Maglietta) share a similar personality and an aesthetic resemblance, with their curly hair and boho-chic style. Tutto Può Succedere also chooses to keep the cultural and ethnic difference between Carlo and his partner Feven (Esther Elisha), just like Crosby and Jasmine (Joy Bryant) in the American version. On a broader level, we see that each one of these details appear more polished in the stylistically conventional Rai remake.
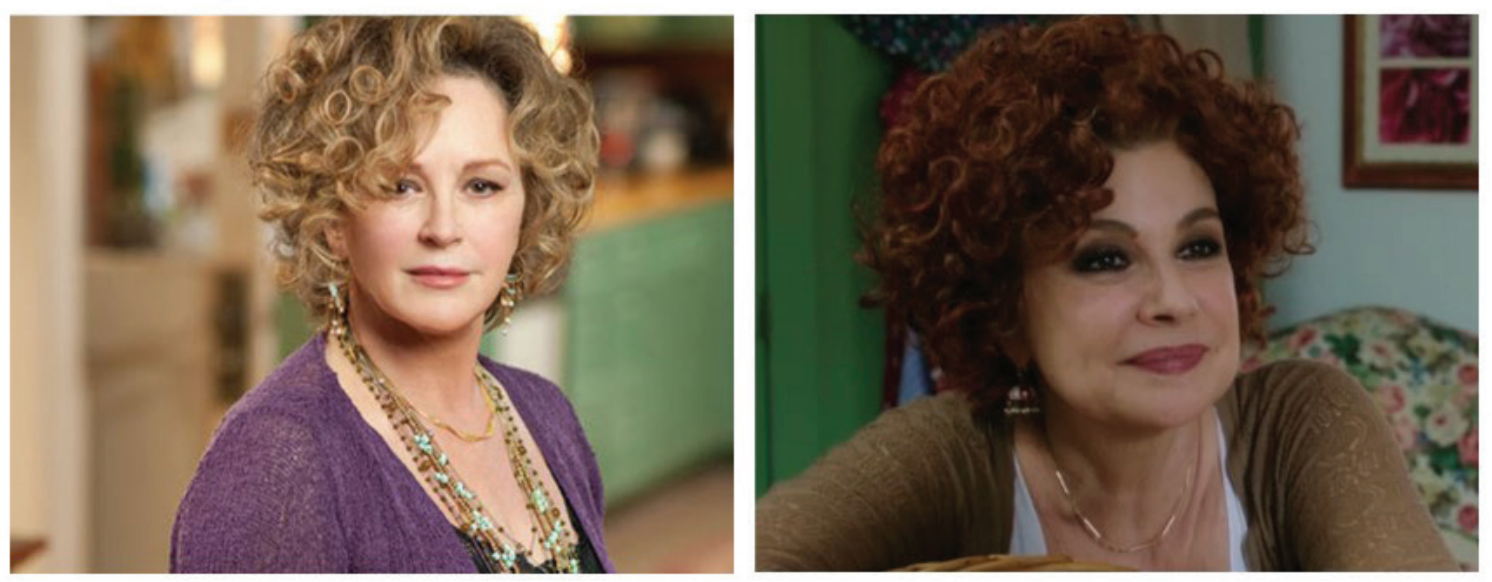

Figure 5. The grandmothers: Camille (Bonnie Bedelia, left) and Emma (Licia Maglietta, right).

The functioning similarity between the two series couldn't have been pursued if it wasn't for a common ground of values, purposes and themes. We can better understand it borrowing the concept of "multiple layers of cultural proximity", ${ }^{16}$ taking into account that "'Culture' in cultural negotiation refers to a wide range of meaning-making processes within production, text, and reception, and includes both material and symbolic aspects": ${ }^{17}$ Parenthood is a family drama, a television genre that is particularly suitable to Italian audiences and specifically to Raiuno's target audience. In the show, the family is represented as a large, diverse group of people, showing different kinds of nuclear units, including single parenting and, further on, divorced parents. However, at its core it is still a patriarchal structure led by grandfather Zeek (Craig T. Nelson)/Ettore (Giorgio Colangeli), where men and women take on traditional gender roles. Such roles are mildly questioned in single mother Sarah/Sara storyline, and through the recurring motherhood vs. career dichotomy, that surfaces in different ways both in Julia (Erika Christensen)-Joel (Sam Jaeger), that is Italian Giulia-Luca, and Kristina-Adam (Cristina-Alessandro) marriages, as we will see further on.

Moreover, the centralizing force represented by the grandparents perfectly adapts to the relevance of grandparenting in Italian culture, where grandparents' presence in raising children is common.

In spite of marital problems and a frequent lack of communication, Zeek-Camille and Ettore-Emma stand as the reassuring cornerstones of the family, equipped with opposite and recognizable personalities: Camille and Emma are sympathetic women, excellent cooks, good listeners, wise advisors; Zeek and Ettore are hot-tempered, rather chauvinist alpha males who struggle to empathize with their spouses and children; nonetheless they are able to occasionally change into better human beings.

Rifts, conflicts and disagreements among family members are regularly recomposed through emotional storylines that tend towards happy endings, or at least towards resolutions and closures. 
As previously asserted, Scott Olson's definition of narrative transparency seems to be well suited to the case of Tutto Può Succedere:

Transparency is the capability of certain texts to seem familiar regardless of their origin, to seem part of one's own culture, even though they have been crafted elsewhere. The commercial advantage to a movie or television program of his type is that it has the potential to garner a large global market. A media product that lacks transparency has much more limited commercial possibilities. ${ }^{18}$

Narrative transparency allows a cultural product to better blend into other cultures, and grants it a competitive advantage over other potentially exportable commodities. Parenthood's sense of familiarity is sustained by topics and plots that resonate with local understandings, and are therefore more easily transposed to Italian public service.

\subsection{Repetition Without Replication: Something Has to Change in Tutto Può Succedere}

Although we have traced ready-made traits characterising the two series, Tutto può succedere needs to differentiate from the original according to local requirements. The differentiation is twofold, with regard to the formal-aesthetic aspects on one level, and to the content on the other. Specifically, the Italian version went through a standardisation process that normalised Parenthood's distinguishing style as well as softened potentially controversial plot elements. This happens taking into account that "divergence/modifications occur at particular moments due to culturally different interpretations of certain contentious issues such as religion, domesticity, sexuality, womanhood, taboos, and social stratification". ${ }^{19}$ Moreover, as Jolien van Keulen argued, the "exchange of knowledge, technologies, ideas and production practices" that characterises transnational television also concerns its aesthetics, "defined as the combination of technical elements of a television programme: cinematography, editing and sound" 20 : as well as narrative preferences and expectations, television aesthetics - such as camera angles, editing choices, and so on - need to be relocated according to culturally determined tastes and standards.

As for the formal-aesthetic aspects, Parenthood is characterised by camera movements, fast paced editing, quick and rich dialogues, substantial and signifying use of camera focus. While Tutto Può Succedere keeps an overall sense of movement that, especially in choral sequences, expresses the messy everyday life of a numerous family, stylistically speaking the series reduces the pace, levels out the richness of the camerawork, and avoids visual and voices overlapping.

When it comes to contents and plot, Tutto Può Succedere resorts to typify its characters in a softened, stereotypical fashion, smoothing and simplifying every debatable topic. Sometimes the Italian version chooses to ignore some plot points or underline others. For instance, Tutto Può Succedere skips details aimed to drive the conversation and to emerge as a cause for reflection for the characters and audience. Here are some emblematic changes or modifications to the storylines.

The age difference between Sarah and the much younger teacher she dates in Parenthood is often addressed by the characters as something both unusual and to joke about, while in Tutto Può Succedere it is barely mentioned, and not so clear in the actors' look, therefore the series loses the opportunity to depict an atypical take on age difference in couples, more commonly represented the other way around.

Sarah is also a single mother of two, who struggles with a difficult past and financial problems. In Parenthood, she unapologetically works as a bartender while taking occasional jobs. In Tutto può succedere Sara finds a job in a 
bookshop, and in the third season in a photographer's studio, choice that sounded probably somehow more acceptable in the mind of the authors, as if Sara's already harsh life needed to be dignified by a more respectable workplace.

Julia and Joel Graham are defined by reverse gender roles: Julia is a career woman and the breadwinner of her family, while Joel is a stay-at-home dad and the main caregiver of their daughter Sydney. In Tutto Può Succedere such features are kept, however, Luca is more troubled than Joel in accepting this arrangement. Luca's reluctance and his consequent arguments with Giulia, as well as Ettore's mockery and misogynistic remarks (he calls him "la donna di casa") reframe the family in a slightly reactionary 'battle of the sexes' vibe that lacks the nuances of the original.

Facing a personal crisis, grandma Camille-Emma decides to get back some space for herself. In Parenthood she turns to painting, in Tutto Può Succedere she joins a tennis club, counterposing an upper class habit to Camille's hobby, that better suits her bohemian, post-hippy style.

It's worth noting that in Tutto Può Succedere issues about sexual health and sex education tend to disappear. At the beginning of the series, Crosby's fear of commitment and responsibility is briefly addressed through his girlfriend's desire to have a baby using IVF, a plot detail that never occurs in Tutto Può Succedere. When Julia's daughter Sydney asks her mom to explain where babies come from, Julia put aside the surprise and explicitly tell her the truth with words suitable for children. When Giulia's daughter Matilde does the same, the Italian series cautiously removes the interesting child-friendly sex education lesson that Julia gave, showing Giulia whispering something inaudible in Matilde's ear. This case in particular illustrates Rai overcautiousness about what their audiences can or cannot bear, and what they can or cannot be bothered about.

Rai's normalisation effort demonstrates the ways Rai interprets the adaptation process, and intends to appeal a generalist audience, smoothing over potentially controversial details. However, two aspects define Tutto Può Succedere and differentiate it from Parenthood.

Firstly, the soundtrack. Parenthood often uses pop and rock classics or contemporary indie music, while Tutto Può Succedere's soundtrack is composed by Italian musicians only. This choice fulfills two sets of purposes. On the one hand, it enhances a sense of national specificity: original score is composed by Paolo Buonvino and performed by Negramaro and Raphael Gualazzi, but the soundtrack also features many songs by Italian singers or bands. On the other hand, we see some Italian bands and singers actually perform diegetically in Carlo's bar (A Toy Orchestra, España Circo Este, Calcutta among others), serving a mutual promotional purposes for both the musicians and the series, appealing the bands' fans and, more broadly, younger audiences.

Secondly, in season one of Tutto Può Succedere Ambra is briefly romantically involved with her schoolmate Giada, with whom she forms a music duo. In Parenthood a same-sex relationship occurs later in season five, and it involves another character, Haddie (Federica in Tutto può succedere). As we previously said, Rai usually plays safe and avoids unconventional plots, therefore addressing an LGBT storyline can be considered an unusually bold choice, especially for the first channel, the most traditional and mainstream of the three PBS channels. However, it is worth noting that, first, it is fair to suspect that switching to Ambra reveals the prejudice that a hint to bisexuality would be more acceptable when referred to the reckless troublemaker of the family. Second, quickly dismissing the storyline lessens the relevance of the topic, while Haddie and Lauren's relationship in Parenthood is more deeply explored.

\section{Conclusion}

Just to give a quick overview about the process of domestication underwent by Parenthood in its Italian remake, we argued that some of the characteristics defining its cultural proximity made it the perfect TV series to adapt for Italian 
audiences. As a matter of fact, the family drama genre is particularly well suited for the Italian culture, as well as for the Italian television programme production tradition, being one of the most popular in the history of Italian TV. Although recalling the entire history of Italian television seriality is not a purpose of this article, we highlight that one of its characteristics has always been a mimetic approach with a routinisation of the stories, tending to familiarisation and reassurance, even when the storytelling deals with dramatic issues. ${ }^{21}$ We can also argue that Italian TV narratives revolve around a few recurring types of storytelling, such as crime/cop drama, biopic, comedy, family drama, and period drama focused on historical events that were crucial to civil society's memory and sensibility. ${ }^{22}$

Moreover, the central role of the family in Italian culture has been recognized by Milly Buonanno:

Apart from the undeniable changes occured in the family structure and sense in the last two or three decades, Italy is the western country where in this peculiar sphere the elements of continuity with the past are manifold and stronger: from the solidity of the family bonds, to the enduring ties, to the extended cohabitation through generations, to the reciprocal support within the family ties. ${ }^{23}$

We can extend this argument underlining that one of the peculiarities of Italian TV series mainstream production is the insertion of family and interpersonal relationships themes in many different TV serial genres, as recalled by Daniela Cardini:

Some prevalent themes emerge from Rai fiction. In contrast to U.S. storytelling, which is based on complex plots where characters' relationships are usually pertinent to the public sphere or to the workplace, Italian plots revolve around domestic issues and interpersonal connections among family members and those closest, related by love, friendship and other forms of relationships that are pertinent to the private sphere. ${ }^{24}$

For this reason, many aspects and themes of the original plot dealing with family bonds, everyday life and events were replicated in Tutto Può Succedere, and they can be conceptualised as ready-made elements that maintain the original template of the original TV series. But following what characterises the average process of format adaptation, the Italian version made some aesthetic and content adjustments. Such changes were strategic not only because they aimed at making the product perceived as 'local', rooted in Italian culture, but also because they responded to Rai goals and strategic targets. Besides the idea of "shielding" a traditional audience (or better to say the perception, the image of a traditional audience) from issues that might be too problematic and divisive, Rai also aimed to get closer to a younger audience who usually avoids Rai television schedule. As a matter of fact, Rai and every other mainstream broadcaster share the problem of the ageing of its audience and the related loss of younger audiences, which therefore becomes a particularly important target to be pursued. Rai chased the goal of engaging such reluctant segment employing various strategies, such as uploading the preview of Tutto Può Succedere's third season on Rai streaming platform Rai Play, one of the first experiments with this mode of release. According to Gian Paolo Tagliavia, Rai chief digital officer, the results were positive, with 2.5 million views collectively. ${ }^{25}$

The appeal to a cross generational audience of Tutto Può Succedere is testified on the one hand by the mixed audience of the series that includes younger segments, as underlined by head of Rai Fiction Eleonora Andreatta; ${ }^{26}$ on the other hand it is confirmed by the protests raised by the unexpected cancellation of the series after the third season, resulting in social media campaigns and even a petition on Change.org to renew the series for a fourth season. The audience numbers fluctuated between 4.2 million viewers and 19\% share in season two and 2.7 million viewers and $14 \%$ share in season three. The cancellation was justified with the low ratings of season three, which aired in summer 2018 and suffered from the competition with the popular show Temptation Island and the 2018 FIFA World Cup.

In conclusion, we believe that in Tutto Può Succedere we are witnessing the realisation of a paradox: on the one hand the normalisation of differences and controversial details, constructing a more traditional storytelling; and on the other hand, the desire to appeal to an audience that, by definition, is untraditional. 


\section{Notes}

1. Albert Moran, New Flows in Global TV (Bristol: Intellect, 2009), 16.

2. Ibid., 17

3. Mats Nylund, "Television Format as a Transnational Production Model", in New Patterns in Global Television Formats, eds. Karina Aveyard, Albert Moran, and Pia Majbritt Jensen (Bristol: Intellect, 2016).

4. Jean K. Chalaby, "Drama without Drama: The Late Rise of Scripted TV Formats," Television \& New Media 17, no. 1 (2016).

5. Albert Moran and Karina Aveyard, "The Place of Television Programme Formats," Continuum. Journal of Media \& Cultural Studies 28, no. 1 (2014): 19.

6. A concept coined by Reg Grundy to describe the characteristics inherent to the marketing, licensing and adaptation of a format across national borders, whose ultimate goal is its customisation in a local version to be recognized as a local production by the audience.

7. Miriam Stehling, "From Localisation to Translocalisation: Audience Reading of the Television Format Top Model," Critical Studies in Television 8, no. 2 (2013).

8. Isadora Garcia Avis, "Adapting Landscape and Place in Transcultural Remakes. The case of Bron/Broen, The Bridge and The Tunnel," Series. International Journal of TV Serial Narratives 1, no. 2 (2015): 130.

9. Joseph Straubhaar, "Beyond Media Imperialism: Asymmetrical Interdependence and Cultural Proximity," Critical Studies in Mass Communication 8, no. 1 (1991).

10. Antonio C. La Pastina and Joseph D. Straubhaar, "Multiple Proximities Between Television Genres and Audiences," Gazette: International Journal for Communication Studies 67, no. 3 (2005).

11. Scott Robert Olson, Hollywood Planet: Global Media and the Competitive Advantage of Narrative Transparency (Mahwah, NJ: Lawrence Erlbaum Associates Inc., 1999), 14.

12. Edward Larkey, Landry Digeon, and Ibrahim Er, "Measuring Transnationalism. Comparing TV Formats Using Digital Tools," VIEW Journal of European Television History \& Culture 5, no. 9 (2016): 4.

13. Authors' translation. Tutto Può Succedere website is currently offline.

14. Larkey et al., "Measuring Transnationalism".

15. Albert Moran and Justin Malbon, Understanding the Global TV Format (Bristol: Intellect, 2006), 6-7.

16. La Pastina and Straubhaar, "Multiple Proximities Between Television Genres and Audiences".

17. Heidi Keinonen, "Television Format as Cultural Negotiation. Studying Format Appropriation Through a Synthesizing Approach," VIEW Journal of European Television History and Culture 5, no. 9 (2016).

18. Olson, Hollywood Planet, 14.

19. Ibrahim Er, "A Funnier Monk: A Multimodal Approach to Transnational TV Series Adaptations," Series. International Journal of TV Serial Narratives 2, no. 1 (2016): 10.

20. Jolien van Keulen, "Aesthetic Proximity. The Role of Stylistic Programme Elements in Format Localisation," VIEW Journal of European Television History and Culture 5, no. 9 (2016).

21. Felicita Gabellieri, "Un Sogno Condiviso. Le Audience di Un Medico in Famiglia" [A Shared Dream. The Audiences of the TV Show Un Medico in Famiglia], in Realtà Multiple. Concetti, Generi e Audience della Fiction TV [Multiple Realities. Concepts, Genres and Audiences of the TV Fiction], ed. Milly Buonanno (Napoli: Liguori, 2004) (authors' translation).

22. Cecilia Penati, "I Generi Televisivi come Pratiche" [Television Genres as Practices], in La Televisione. Modelli Teorici e Percorsi d'Analisi [Television. Theoretical Models and Pathways of Analysis], eds. Massimo Scaglioni and Anna Sfardini (Roma: Carocci, 2017).

23. Milly Buonanno, Leggere la Fiction. Narrami o Diva Rivisitata [Reading the Fiction. Tell Me o Goddess Revisited] (Napoli: Liguori, 1996), 211.

24. Daniela Cardini, La Lunga Serialità Televisiva: Origini e odelli [The Long-Lasting TV Seriality: Origins and Models] (Roma: Carocci, 2004), 48.

25. See "Rai: Successo Dell'anteprima Su Rai Play Della Nuova Serie Di Tutto Può Succedere," Rai Ufficio Stampa, June 18, 2018, https://www.rai.it/ufficiostampa/assets/template/us-articolo.html?ssiPath=/articoli/2018/06/SUCCESSODELLANTEPRIMA-SU-RAI-PLAY-DELLA-NUOVA-SERIE-DI-TUTTO-PUO-SUCCEDERE-27a4fffc-8ee5-443b-9432690255d517b7-ssi.html

26. See "Rai, Andreatta: Tutto Può Succedere Ha Vinto La Sfida Estiva," Rai Ufficio Stampa, August 7, 2018, https://www.rai.it/ ufficiostampa/assets/template/us-articolo.html?ssiPath=/articoli/2018/08/RAI-ANDREATTA-TUTTO-PUO-SUCCEDEREHA-VINTO-LA-SFIDA-ESTIVA-c262a4c6-e22a-431c-b535-9b314b747f14-ssi.html 


\section{Biographies}

Stefania Antonioni is associate professor at the Department of Communication, Humanities and International Studies of the University of Urbino Carlo Bo, where she teaches Cinema and Photography, Advertising Theories and Strategies of Promotional Communication. Her main research interests are moving image studies, visual studies, branded entertainment and promotional strategies. Among her publications Imagining. Serialità, narrazioni cinematografiche e fotografia nella pubblicità contemporanea (2016) and with M. Farci Post-millennial spectatorship and horror films: The case of It (2018).

Chiara Checcaglini completed her Ph.D. in Sociology and Communication Studies at the University of Urbino Carlo Bo. Her current research interests include media education, transmedia storytelling, film criticism, contemporary serial narratives, their critical reception and their forms of distribution. She wrote essays and presented papers on these topics and she authored the book Breaking Bad. La chimica del male: Storia, temi, stile (Mimesis 2014). She is teaching assistant of Cinema and Photography at the University of Urbino and she has taught Media Literacy and Transmedia Storytelling at the University of Udine. She contributes to II Cinema Ritrovato Film Festival and she is editor and contributor of several webzines dedicated to film and television criticism. 